\title{
Computed tomography surveillance helps tracking COVID-19 outbreak
}

\author{
Akihiro Machitori ${ }^{1} \cdot$ Tomoyuki Noguchi $^{2,3,4}{ }^{1} \cdot$ Yusuke Kawata $^{1} \cdot$ Nobuhiko Horioka $^{5} \cdot$ Akihiro Nishie $^{6}$. \\ Daisuke Kakihara $^{6} \cdot$ Kousei Ishigami $^{6} \cdot$ Shigeki Aoki $^{7} \cdot$ Yutaka Imai $^{8}$
}

Received: 5 June 2020 / Accepted: 26 July 2020 / Published online: 7 August 2020

(c) Japan Radiological Society 2020

\begin{abstract}
Purpose To reveal that a computed tomography surveillance program (CT-surveillance) could demonstrate the epidemiologic features of COVID-19 infection and simultaneously investigate the type and frequency of CT findings using clinical CT data. Materials and methods We targeted individuals with possible CT findings of viral pneumonia. Using an online questionnaire, we asked Japanese board-certified radiologists to register their patients' information including patient age and sex, the CT examination date, the results of PCR test for COVID-19 infection, CT findings, and the postal code of the medical institution that performed the CT. We compared the diurnal patient number and the cumulative regional distribution map of registrations in CT-surveillance to those of the PCR-positive patient surveillance (PCR-surveillance).

Results A total of 637 patients was registered from January 1 to April 17, 2020 for CT-surveillance. Their PCR test results were positive ( $n=62.5-398 \%)$, negative $(n=8.9-57 \%)$, unknown $(n=26.2-167 \%)$, and other disease $(n=2.4-15 \%)$. An age peak at 60-69 years and male dominance were observed in CT-surveillance. The most common CT finding was bilaterally distributed ground-glass opacities. The diurnal number and the cumulative regional distribution map by CT-surveillance showed tendencies that were similar to those revealed by PCR-surveillance.
\end{abstract}

Conclusion Using clinical CT data, CT-surveillance program delineated the epidemiologic features of COVID-19 infection.

Keywords COVID-19 $\cdot$ Pneumonia $\cdot$ Viral $\cdot$ Tomography $\cdot$ X-ray computed $\cdot$ Surveys and questionnaires $\cdot$ Public health practice

Tomoyuki Noguchi

tnogucci@radiol.med.kyushu-u.ac.jp

Akihiro Machitori

dmachitori@hospk.ncgm.go.jp

Yusuke Kawata

dkawata@ hospk.ncgm.go.jp

Nobuhiko Horioka

horioka-nobuhiko@mhlw.go.jp

Akihiro Nishie

anishie@ radiol.med.kyushu-u.ac.jp

Daisuke Kakihara

kakky@radiol.med.kyushu-u.ac.jp

Kousei Ishigami

ishigami@ radiol.med.kyushu-u.ac.jp

Shigeki Aoki

saoki@juntendo.ac.jp

Yutaka Imai

imaiy@is.icc.u-tokai.ac.jp

1 Department of Radiology, National Center for Global Health and Medicine, Kohnodai Hospital, 1-7-1 Kohnodai,

Ichikawa City, Chiba Province 272-8516, Japan
2 Department of Clinical Research, Center for Clinical Sciences, National Center for Global Health and Medicine, 1-21-1 Toyama, Shinjuku-ku, Tokyo 162-8655, Japan

3 Department of Radiology, National Hospital Organization Kyushu Medical Center, 1-8-1 Jigyohama, Chuo-ku, Fukuoka City, Fukuoka Province, Japan

4 Department of Clinical Research, National Hospital Organization Kyushu Medical Center, 1-8-1 Jigyohama, Chuo-ku, Fukuoka City, Fukuoka Province 810-8563, Japan

5 General Affairs Division, Health Policy Bureau, Ministry of Health, Labour and Welfare, 1-2-2 Kasumigaseki, Chiyoda-ku, Tokyo 100-8916, Japan

6 Department of Clinical Radiology, Graduate School of Medical Sciences, Kyushu University, 3-1-1 Maidashi, Higashi-ku, Fukuoka City, Fukuoka Province 812-8582, Japan

7 Department of Radiology, Juntendo University, 2-1-1 Hongo, Bunkyo-ku, Tokyo 113-8421, Japan

8 Department of Radiology, Tokai University Hachioji Hospital, 1838 Ishikawa-cho, Hachioji City, Tokyo 192-0032, Japan 


$\begin{array}{ll}\begin{array}{l}\text { Abbreviations } \\ \text { JRS }\end{array} & \begin{array}{l}\text { Japan Radiological Society } \\ \text { CT }\end{array} \\ \text { PCR } & \begin{array}{l}\text { Pomputed tomography } \\ \text { CT surveillance }\end{array} \\ & \begin{array}{l}\text { Computed Tomography Imaging Sur- } \\ \text { veillance of Viral Pneumonia }\end{array} \\ \text { PCR surveillance } & \begin{array}{l}\text { PCR-positive patient surveillance pro- } \\ \text { vided by Japan's Ministry of Health, }\end{array} \\ \text { RT-PCR } & \begin{array}{l}\text { Labor and Welfare } \\ \text { Reverse transcription-polymerase } \\ \text { chain reaction }\end{array} \\ \text { GGO } & \begin{array}{l}\text { Ground-glass opacity } \\ \text { Ministry of Health, Labour and }\end{array} \\ \text { MHLW } & \text { Welfare } \\ \text { mSv } & \text { Millisieverts }\end{array}$

Introduction

With the outbreak of an unidentified pneumonia in Wuhan, China in December 2019, millions of people are now aware of the rage of the COVID-19 pandemic that continues to spread. Here in Japan, a state of emergency was declared on April 7, 2020 for the prevention and control of COVID-19 infections. To respond as quickly as possible to the dynamically changing crisis of the rapid spread of COVID-19 infection, it is necessary to gather all of the available information and analysis results regarding COVID-19, in order to formulate the proper research strategies and public health practices to control and prevent COVID-19 infection.

At present, because the most reliable basis for the diagnosis of COVID-19 is a positive result on a reverse transcription-polymerase chain reaction (RT-PCR) test, the surveillance of the PCR-positive patients for epidemiological features (hereinafter referred to as PCR surveillance) is ongoing. Compared to monitoring for suspected carriers, PCR surveillance has the advantage of revealing the trends of COVID-19 infection more accurately. However, PCR surveillance has the disadvantages of low sensitivity and inconsistent results [1]. There is also a delay of one to several days until the results of the PCR test for COVID-19 are known. In addition, the current policy of PCR testing limited to symptomatic patients in Japan makes it difficult to track individuals who are in the incubation period and those who are asymptomatic carriers.

COVID-19 pneumonia shows characteristic features on computed tomography (CT) [1-10], and its CT findings are similar to those of viral pneumonia in general, such as influenza pneumonia. We speculated that it might be possible to detect individuals with suspected COVID-19 pneumonia by CT, even without making a definitive diagnosis. We thus launched a surveillance program based on CT examination findings. We call the program 'Computed Tomography
Imaging Surveillance of Viral Pneumonia,' (hereinafter referred to as CT surveillance) as it is designed to identify suspected or confirmed COVID-19 carriers with CT findings that are similar to those of COVID-19 pneumonia. This CT surveillance program uses an online questionnaire form as one of the projects of the Japan Safe Radiology Conference to ensure the quality and safety of radiology [11]. We analyzed the registration data and here reported the interesting results of the CT surveillance. We conducted the present study to reveal that CT-surveillance could demonstrate the epidemiologic features of COVID-19 infection as well as simultaneously investigate the type and frequency of characteristic imaging findings on CT by using clinical CT data.

\section{Materials and methods}

\section{Study design}

This study was approved by the Ethics Review Committee of the Japan Radiological Society (JRS) on March 1, 2020, waiving the need for written informed consent from the patients.

\section{Surveillance design}

\section{Questionnaire entries}

We targeted patients who showed the possible CT findings of viral pneumonia, and we started the patient registration on March 5, 2020. We determined the period for targeted patients as January 1, 2020 and thereafter. The time reference was set to the date when the individual's first CT examination was performed, rather than the medical examination day or the onset of symptoms. The patient's gender and age at the time of the CT scan were registered. The PCR test for COVID-19 infection was used as the basis of the clinical diagnosis, and the test results were classified as one of the following four categories: positive, negative, or unknown for COVID-19 infection, or other disease. The characteristics of the CT findings were as follows: no findings, bilateral lung involved, a unilateral lung involved, ground-glass opacity (GGO) referring to an area of increased attenuation with preserved vessels and bronchial structures, crazy-paving pattern (grounds with reticular shadows) referring to an area of scattered or diffuse ground-glass attenuation with superimposed interlobular septal thickening and intralobular septal thickening, and consolidation (infiltrative shadow) referring to an area of the alveolar airspaces being replaced with fluid. Multiple findings were possible (Table 1).

As the site of infection, instead of the patient's address or the address of the patient's medical institution both of which are officially concealed from publicity, the postal code of 
Table 1 Contents of the questionnaire for CT surveillance

\begin{tabular}{ll}
\hline No & Items \\
\hline 1 & Date of CT examination \\
2 & Patient age at the time of CT \\
3 & Patient sex \\
4 & PCR test for COVID-19 infection: \\
& Positive \\
& Negative \\
& Unknown \\
& Other disease \\
& CT findings (multiple answers allowed): \\
5 & No findings \\
& Bilateral lung \\
& Unilateral lung \\
& Ground-glass opacity \\
& Crazy-paving pattern \\
& Consolidation \\
& Postal code of the medical institution performing CT \\
& Registration number of the board-certified radiologist \\
\hline
\end{tabular}

the medical institution where the CT images were taken was registered. The registration number of the board-certified radiologist and his/her name were requested for entry as proof of the registrant.

\section{The support from JRS}

This study had generous support from JRS. The online questionnaire system was opened on the member website of JRS and Japan College of Radiology (JCR). Using their email lists, JRS and JCR sent out messages to their members requesting their patients' registration in the system, and JRS sent a notification by postal mail to each of the trainingdesignated medical institutions of Japan to invite a single, representative radiologist of each institution to the current surveillance program. JRS managed both the transfer of the information that was obtained to Japan's Ministry of Health, Labour and Welfare (MHLW) and the online publication of the questionnaire on JRS and JCR member websites.

\section{Data analysis}

We compared the diurnal numbers of patient registrations in CT surveillance program with those of the PCR surveillance program, which were announced by MHLW as a referential standard [12]. For this comparison, we used the Spearman signed rank test and a Bland-Altman analysis for the interchangeability of the two surveillance programs by plotting the differences between the two against the averages of the two. The cumulative regional distribution map obtained by
CT surveillance was visually compared to that obtained by PCR surveillance. We also analyzed CT findings, age distribution, gender differences, and clinical diagnoses obtained by CT surveillance. In addition, differences in CT findings between the PCR-positive and -negative patients were evaluated to clarify the priority of CT findings for early COVID19 pneumonia using a chai-square test. All statistical analyses were evaluated with a significance level of 0.05 .

\section{Results}

A total of 637 individuals who underwent a CT examination during the approximately 3.5-month period from January 1 to April 17, 2020 were registered in CT surveillance program during the period of March 5 to April 18, 2020. There were 408 (64\%) males and 229 (36\%) females, which is approximately 1.8 times more males than females. The averaged ages of the males and females were 59.5 and 58.1 years old, respectively. The 637 subjects' results on the PCR test for COVID-19 or other diseases in the clinical diagnosis were as follows. Positive: $n=398(62.5 \%)$, negative: $n=57$ (8.9\%), unknown: $n=167$ (26.2\%), and other disease: $n=15$ $(2.4 \%)$. Patients with other disease were recorded as having the influenza pneumonia $(n=6)$, the bacterial pneumonia $(n=5)$, the pneumocystis pneumonia $(n=3)$, and the acute interstitial pneumonia $(n=1)$.

In contrast, PCR surveillance conducted by Japan's MHLW during the same 3.5-month period from January 1 to April 17, 2020 accumulated a total of 9,654 patients.

The age distribution in the CT surveillance peaked at 60-69 years, and a substantially high incidence was observed at 40-89 years. The number of subjects aged 20-29 or 30-39 years old was less than one-half the number of subjects aged 60-69 years old, and the number of subjects 0-19 or 90-99 years old was less than $10 \%$ of those aged 60-69 years old (Fig. 1).

The epidemic curve of the diurnal patient number in CT surveillance showed a distribution that was similar to that of PCR surveillance (Figs. 2a, b), with a significant correlation (Fig. 2c, $p<0.001$ ). However, there was a proportional error between the two surveillance programs' diurnal patient numbers indicated by the Bland-Altman analysis (Fig. 2d), which indicated that the number of patients registered in CT surveillance did not agree with the number of patients registered in PCR surveillance in proportion to the increase in the number of the PCR-positive patients. Meanwhile, our visual inspection revealed that both the CT and PCR surveillance programs showed the outbreak of the epidemic, that is, the increased number of patients sharply (arrows in Fig. 2a, b) after the several consecutive Japanese holidays in midMarch (caps in Fig. 2a, b). 


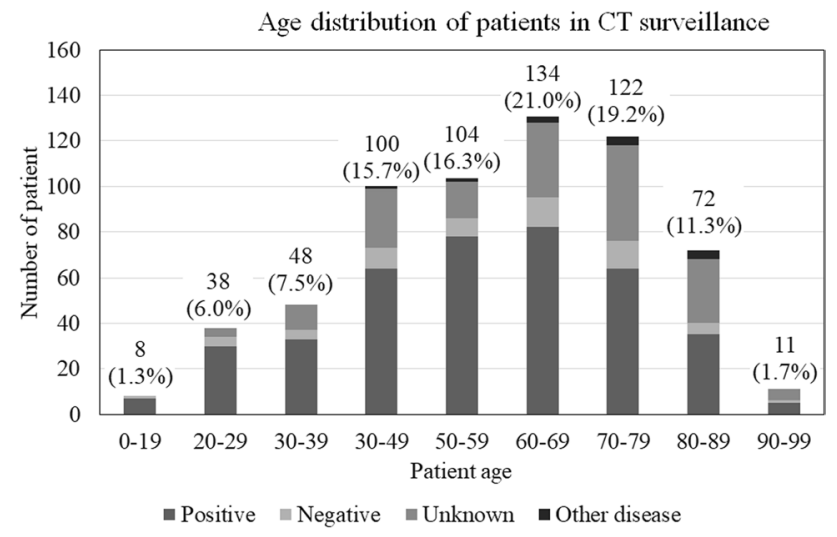

Fig. 1 The age distribution in the CT surveillance peaks at 60-69 years, and a substantially high incidence was at 40-89 years. The numbers of subjects aged 20-29 and 30-39 years old were less than one-half of those 60-69 years old, and the numbers of subjects aged $0-19$ and $90-99$ years were less than $10 \%$ of the number of subjects aged 60-69 years old

The cumulative regional distribution map provided by CT surveillance (Fig. 3a) was similar to that provided by PCR surveillance (Fig. 3b). However, no patient was registered in some of Japan's 47 prefectures in CT surveillance.

The most common CT findings judged by radiologists were bilateral lung (male: female $=78.2-82.4 \%$ ) and distributed GGOs (male: female $=80.8-83.0 \%$ ). The crazy-paving pattern (male: female $=41.0-43.1 \%$ ) and consolidation (male: female $=33.2-35.0 \%$ ) were observed at about onehalf the frequency of GGOs (Fig. 4). Significant differences between the PCR-positive and -negative patients in CT-surveillance were observed regarding bilateral lung involvement $(84.2 \%, 70.2 \%, p<0.01)$ and consolidation $(33.9-50.9 \%$, $p<0.05)$, whereas GGOs (83.5-83.9\%) and the crazy-paving pattern (36.8-44.5\%) did not show a statistically significant difference between the two groups (Fig. 5).

\section{Discussion}

Chest CT has attracted attention as an examination showing high sensitivity to COVID-19 infection [1-10]. However, all of the previous studies other than ours were conducted only on prescreened patients. One of the main reasons for this is that the radiation exposure provided by a CT examination increases the risk of cancer death. The indications for a CT examination should be considered very carefully [13]. In the meantime, the valuable CT data about COVID-19 obtained despite posing the risk to patients should be used effectively, focusing on the welfare of not only the patients but also society at large. JRS is overseeing the Japan Safe Radiology program to ensure the quality and safety of radiology [11].
CT surveillance described herein is being conducted as one of the Japan Safe Radiology projects.

The government's greatest concerns are whether large clusters of medical disruption will occur and how to rescue as many sick patients as possible [14]. It is necessary to identify and monitor the numbers of patients, the expected numbers of patients whose conditions will become severe, and the medical system that can accept patients, and our CT surveillance program might be valuable. Therefore, close cooperation was established in advance with the MHLW regarding the contents of the present surveillance.

When we designed the present investigation, we followed the basic principles of surveillance; that is, the basis of surveillance is 'person, place, and time' [15]. In addition, the practicability, uniformity, and rapidity of the surveillance are more important than complete accuracy [16]. We asked radiologists with regular clinical duties to register patients in the CT surveillance program so that we could carefully select the number of input data needed to effectively conduct the surveillance. The location information that we used was the postal code rather than a physical address, providing the location down to the municipality level in consideration of privacy issues and to prevent harmful rumors.

We observed clear trends of patient gender and age distributions. The age distribution in the CT surveillance peaks at 60-69 years. This tendency might correspond to the degree of the rate of visits to medical institutions due to the severity of symptoms [9]. Our observation of the gender ratio of about 1.8 times more males than females might reflect a tendency of males to be more susceptible to COVID-19 infection and/or a tendency for their conditions to become more severe [17]. These epidemiological propensities merit further investigations of the pathogeny of COVID-19.

Our analyses revealed that the epidemic curve of the diurnal patient number obtained by CT surveillance had a distribution that was similar to that obtained by PCR surveillance. This might be because, in Japan, the testing for COVID-19 infection has been conducted only in patients with officially designated symptoms or close contact with infected persons, and thus the PCR test and CT examination were often performed as a set. Since the result of the PCR test is not known on the day the test is administered, CT surveillance might have the potential to identify the infection on the examination day, assuming that the results are ideally registered as quickly as possible by radiologists throughout the country.

However, we realized that the increase in the patient number in CT surveillance did not well reflect the increase in the patient number in PCR surveillance as uncovered by the Bland-Altman analysis. Although further assessments are required to address the present findings, this result might indicate that (1) the presentation of too great a number of patients discouraged their registration with radiologists, (2) the examining physicians had refined triage skills, and/or (3) 

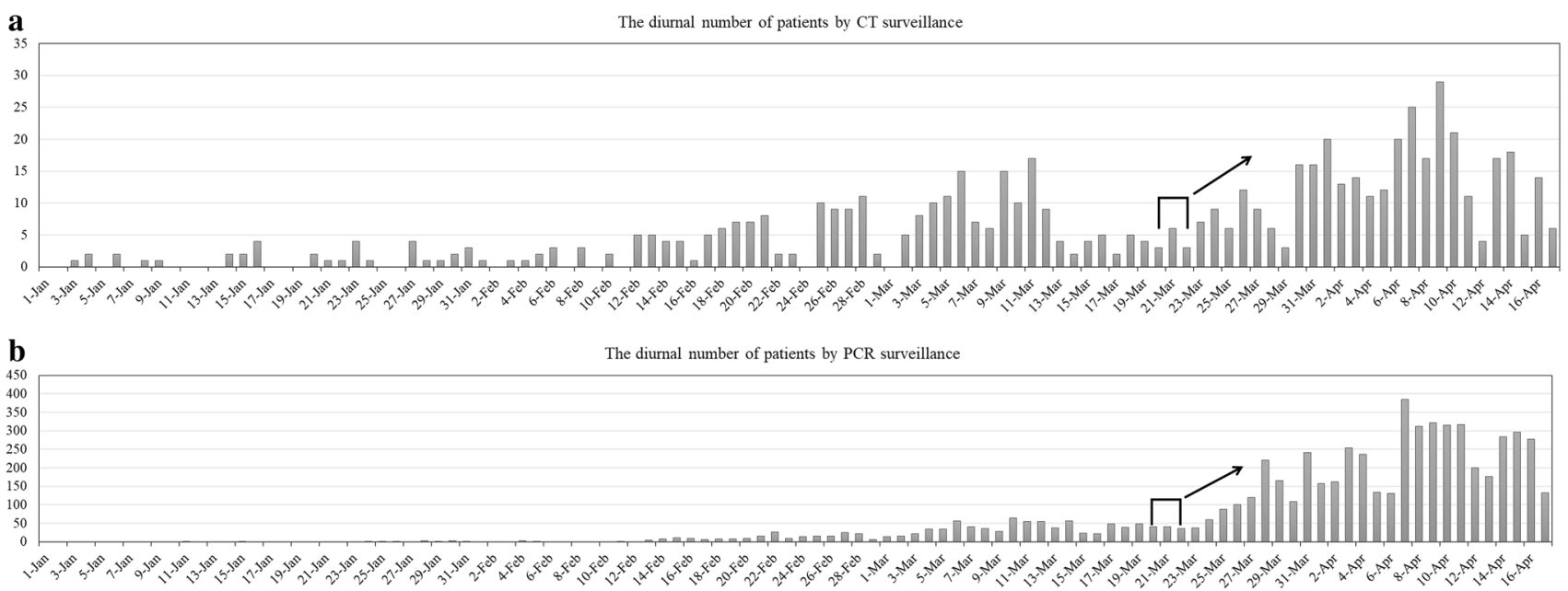

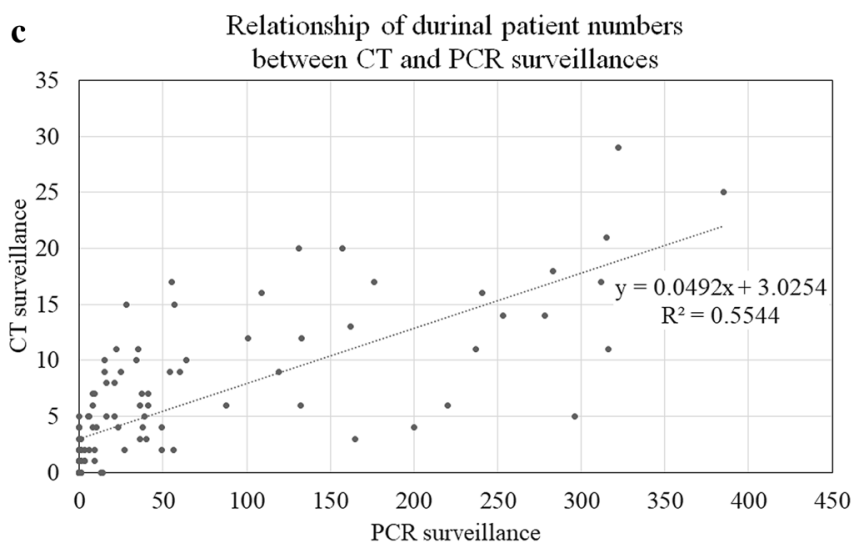

Fig. 2 The epidemic curve of the diurnal patient number in the CT surveillance (a) shows a distribution similar to that of the PCR surveillance (b). Both the CT surveillance and the PCR surveillance revealed the Japanese outbreak of the epidemic, that is, the number of patients increased sharply (arrows in panels $\mathbf{a}$ and $\mathbf{b}$ ) after the consecutive Japanese holidays in mid-March (caps in $\mathbf{c}$ and d). A signifi-

the number of patients exceeded the capacity of the facility for prompt CT examinations.

Our analyses also revealed that the epidemic curve of the diurnal patient number obtained by the CT surveillance, as well as that obtained by the PCR surveillance, reflected the outbreak of the epidemic in Japan, that is, a remarkable increase in the number of patients occurred after the consecutive Japanese holidays in mid-March. The present results indicate that our CT surveillance program could be effective as a tool to grasp the trend of COVID-19 infection.

The cumulative regional distribution map shown by CT surveillance was visually similar to that shown by the PCR surveillance, which might indicate that the publicity about CT surveillance was successful under the generous assistance of the JRS. However, there was a paucity of data from some of the 47 prefectures in Japan. There may also have been radiologists who could not participate in the CT

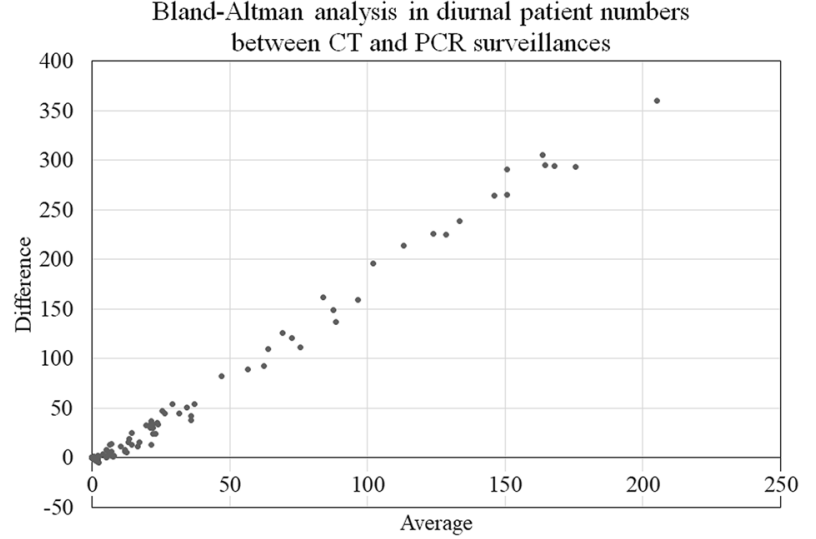

cant correlation between the two surveillance programs was observed $(p<0.001)(\mathbf{c})$. However, the Bland-Altman analysis showed a proportion error (d), suggesting that the number of patients registered in CT surveillance does not rise along with that in PCR surveillance in proportion to the increase in the number of the PCR-positive patients

surveillance for various reasons. As in many countries, there are regional differences in the numbers of radiologists across Japan. In light of the factors described above, a more detailed explanation of the importance of CT surveillance and a larger-scale campaign are necessary in order to make the CT surveillance program as thorough and widespread as possible.

Bilaterally distributed GGOs were the most common CT finding reported by the radiologists in this study, and this is consistent with the previous reports on COVID-19 pneumonia [1-10]. This CT finding is commonly seen in viral pneumonia, and the radiologists who participated in the present study might be highly familiar with CT findings for COVID-19 pneumonia. Besides, consolidation as one of CT findings for COVID-19 pneumonia is reported to be frequent in severe patients [6]. CT findings in CT surveillance might distinguish the group that is considered 
a

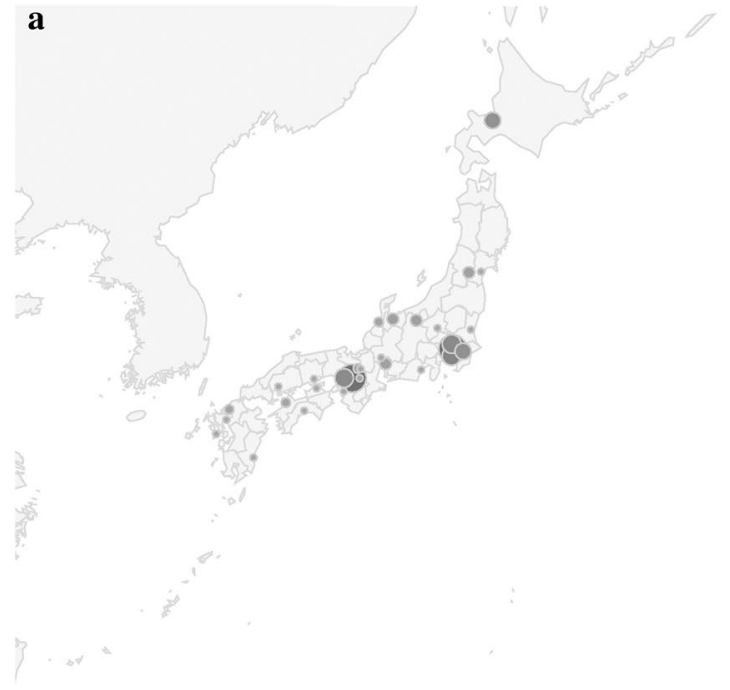

b

Fig. 3 The cumulative regional distribution map obtained by the CT surveillance (a) is similar to that obtained by the PCR surveillance (b). However, there is a paucity of data in some prefectures in the CT surveillance (a)

Fig. 4 The most common CT finding judged by radiologists was bilaterally distributed ground-glass opacities (GGOs). The crazy-paving pattern and consolidation were observed at approximately one-half the frequency of GGOs
Fig. 5 Significant differences between the PCR-positive and -negative patients in CTsurveillance were observed in the bilateral lung involvement $(70.2-84.2 \%)$ and the consolidation (33.9-50.9\%). In the meantime, GGOs (83.5-83.9\%) and the crazy-paving pattern (36.8-44.5\%) were commonly observed

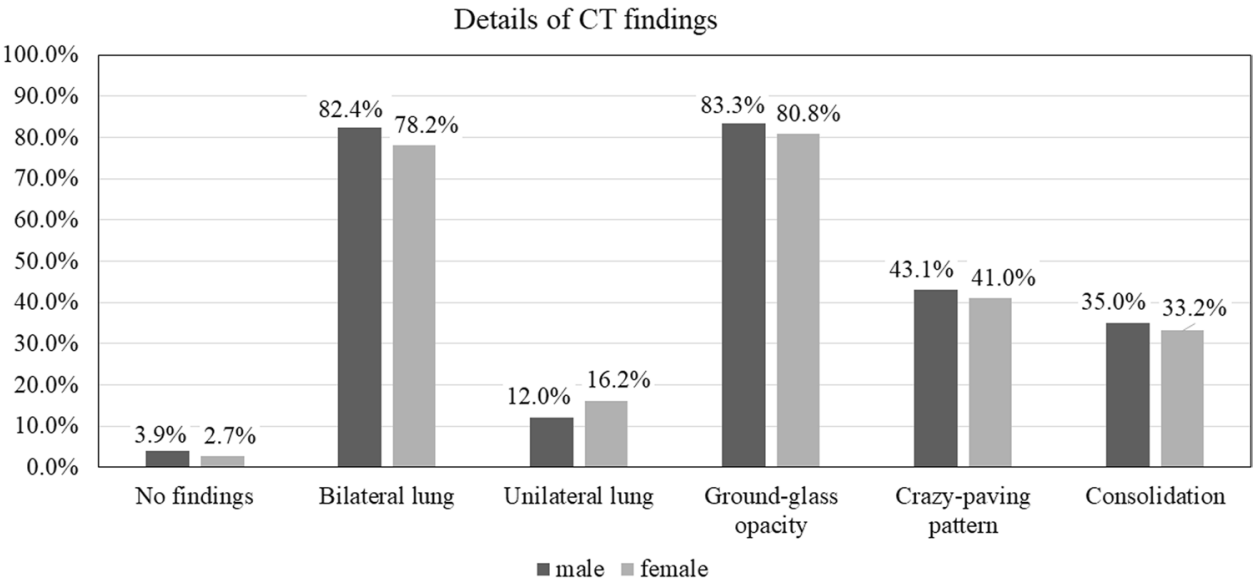

Difference of CT finding between

PCR-positive and negative patient in CT-surveillance

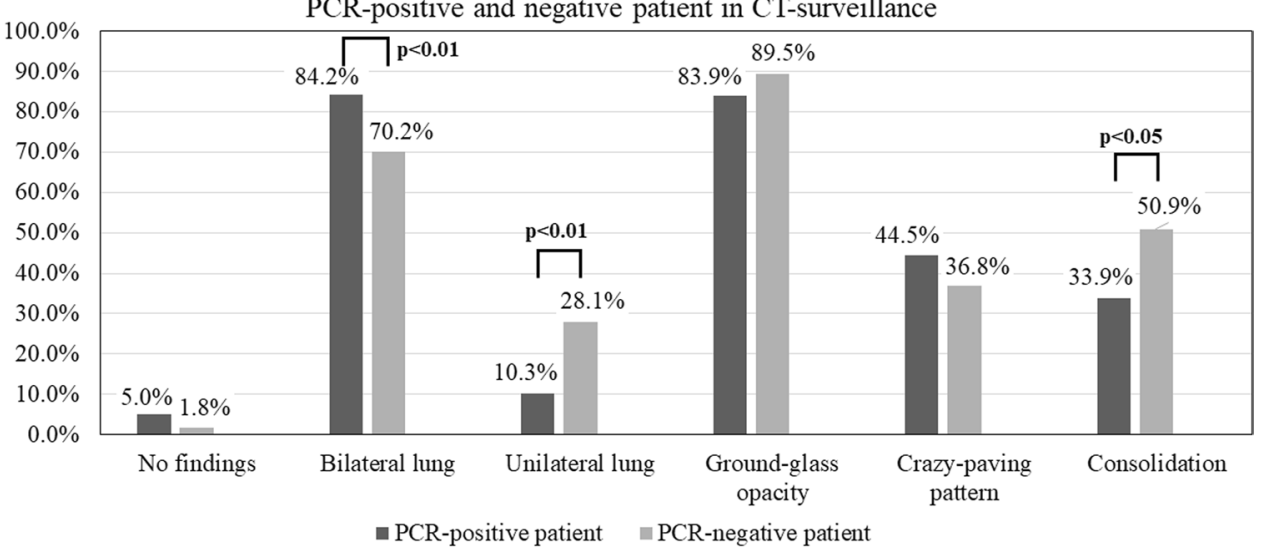


to be mild patients with no findings, GGO, or the crazypaving pattern, from the group that is considered to be severe patients with consolidation, which may be possible to roughly determine the number of patients who get medical treatment at home or at a hotel and those who need hospitalization. However, as drawbacks, the data acquired by CT surveillance is not enough to make a judgment to more accurately divide patients into the mild and severe groups because combining the ratio of the lesion area in the total lung volume and the blood oxygen saturation might be needed [7]. In addition, the PCR test result was not positive for approximately one-third of the registered patients. This might be due to a contamination risk from registering diseases other than COVID-19. However, we clarified the priority of CT findings for early COVID-19 pneumonia by using CT findings of the PCR-positive and -negative patients acquired by CT-surveillance. The PCRpositive patients tend to have the bilateral lung involvement and less frequently had the consolidation on CT compared with the PCR-negative patients.

The current CT surveillance program has several limitations. (1) CT examinations were probably performed only for patients with officially designated symptoms or those who had been in close contact with an infected person. Therefore, our results do not deny the guideline that CT should not be used as a first-line test to diagnose COVID-19 [18]. Further investigations are needed to determine whether or not chest $\mathrm{CT}$ as a first screening test is beneficial, under a careful consideration of the risks of the radiation exposure. (2) There is no uniform and strict standard for CT findings for viral pneumonia. Contamination from other findings is thus a concern. However, the CT surveillance program described herein would be sufficient for the purpose of the collection of suspected COVID-19 carriers' data. (3) The past data of patients with viral pneumonia on CT could be also registered in the current CT surveillance system. Since the real-time registration has not been enforced, and the full shape of the infection curve will be clear only after the COVID-19 outbreak is over [19].

\section{Conclusions}

As one of the projects of the Japan Safe Radiology program, the CT surveillance program were practiced to ensure the quality and safety of radiology. CT and PCR surveillances obtained similar diurnal numbers and regional maps. An age peak at 60-69 years and male dominance were observed in CT surveillance. The most common CT finding was bilaterally distributed ground-glass opacities. CT surveillance delineated the epidemiologic features of COVID-19 infection.
Acknowledgements This work received generous support from the Japan Radiological Society.

Author contributions AM; Project administration, TN; Corresponding author, data analysis, YK; Programming, data analysis, NH; Health Technology Coordinator of the MHLW, AN; data analysis, DK; data analysis, KI; Supervision, SA; Supervision, YI; Supervision.

Funding No funding.

\section{Compliance with ethical standards}

Conflicts of interest The authors wish to confirm that there are no known conflicts of interest associated with this publication and there has been no significant financial support for this work that could have influenced its outcome.

Ethical statement This study was approved by the Ethics Review Committee of the Japan Radiological Society (JRS), which waived the need for written informed consent from the patients.

\section{References}

1. Fang $\mathrm{Y}$, Zhang $\mathrm{H}, \mathrm{Xie} \mathrm{J}$, Lin M, Ying L, Pang P, et al. Sensitivity of chest CT for COVID-19: comparison to RT-PCR. Radiology. 2020;2:432.

2. Ai T, Yang Z, Hou H, Zhan C, Chen C, et al. (COVID-19) in China: a report of 1014 cases. Radiology. 2019;2020:200642.

3. Shi H, Han X, Jiang N, Cao Y, Alwalid O, Gu J, et al. Radiological findings from 81 patients with COVID-19 pneumonia in Wuhan, China: a descriptive study. Lancet Infect Dis. 2020;20(4):425-34.

4. Inui S, Fujikawa A, Jitsu M, Kunishima N, Watanabe S, Suzuki Y, et al. Chest CT findings in cases from the cruise ship "Diamond Princess" with coronavirus disease 2019 (COVID-19). Radiol Cardio Imaging. 2020;2(2):e200110.

5. Zhao W, Zhong Z, Xie X, Yu Q, Liu J. Relation between chest CT findings and clinical conditions of coronavirus disease (COVID19) pneumonia: a multicenter study. Am J Roentgenol. 2020;1:6.

6. Liu KC, Xu P, Lv WF, Qiu XH, Yao JL, Gu JF, et al. CT manifestations of coronavirus disease-2019: a retrospective analysis of 73 cases by disease severity. Eur J Radiol. 2020;126:108941.

7. Yang R, Li X, Liu H, Zhen Y, Zhang X, Xiong Q, et al. Chest CT severity score: an imaging tool for assessing severe COVID-19. Radiol Cardio Imaging. 2020;2(2):e200047.

8. Wen Z, Chi Y, Zhang L, Liu H, Du K, Li Z, et al. Coronavirus Disease 2019: initial detection on chest ct in a retrospective multicenter study of 103 Chinese subjects. Radiol Cardio Imaging. 2020;2(2):e200092.

9. Huang C, Wang Y, Li X, Ren L, Zhao J, Hu Y, et al. Clinical features of patients infected with 2019 novel coronavirus in Wuhan. China Lancet. 2020;395(10223):497-506.

10. Simpson S, Kay FU, Abbara S, Bhalla S, Chung JH, Chung M, et al. Radiological society of North America expert consensus statement on reporting chest CT findings related to COVID-19. RSNA J Imaging. 2020;2(2):e200152.

11. Kakihara D, Honda H, Abe O, Jinzaki M, Tomiyama N, Gobara H, et al (2019) Japan Safe Radiology 2019. ECR 2020 EuroSafe Imaging Poster Exhibition in European Plate Observing System (EPOS). (https://epos.myesr.org/esr/viewing/index.php?modul e=viewing_poster\&task=\&pi=150912). Accessed 17 Apr 2020.

12. The Ministry of Health Labour and Welfare (2020) The situation of occurrence of patients with COVID-19 (map). MHLW 
website. (https://mhlw-gis.maps.arcgis.com/apps/opsdashboard/ index.html\#/0c5d0502bbb54f9a8dddebca003631b8). Accessed 17 Apr 2020

13. International Commission on Radiological Protection. ICRP publication 103. Ann ICRP. 2007;37(24):2.

14. The Ministry of Health Labour and Welfare (2020) About coronavirus disease 2019 (COVID-19). MHLW website. (https://www. mhlw.go.jp/stf/seisakunitsuite/bunya/newpage_00032.html). Accessed 17 Apr 2020.

15. Byass P. Person, place and time-but who, where, and when? Scand J Public Health. 2001;29:84-6.

16. Bonita R, Beaglehole R, Kjellström T. Basic epidemiology. 2nd ed. Switzerland: World Health Organization Press; 2006.

17. Zhou F, Yu T, Du R, Fan G, Liu Y, Liu Z, et al. Clinical course and risk factors for mortality of adult inpatients with COVID19 in Wuhan, China: a retrospective cohort study. Lancet. 2020;395(10229):1054-62.
18. American College of Radiology (2020) ACR recommendations for the use of chest radiography and computed tomography (CT) for suspected COVID-19 infection. ACR website. (https://www.acr. org/Advocacy-and-Economics/ACR-Position-Statements/Recom mendations-for-Chest-Radiography-and-CT-for-Suspected-Covid 19-Infection). Accessed 17 Apr 2020.

19. Centers for Disease Control and Prevention (2013) Interpretation of Epidemic (Epi) Curves during Ongoing Outbreak Investigations. CDC website. https://www.cdc.gov/foodsafety/outbreaks/ investigating-outbreaks/epi-curves.html. Accessed 17 Apr 2020.

Publisher's Note Springer Nature remains neutral with regard to jurisdictional claims in published maps and institutional affiliations. 